\title{
ESTIMATION OF PATCH ARRAY COUPLING MODEL THROUGH RADIATED FIELD MEASUREMENTS
}

M. Sicrua Castañer , J.L. Masa, O. Moreno, J.M. Fernández, D. Castellanos

Abstract: In the desjgn process of aray antennas, coupling is one of the most importan elcments to be counted. The real feeding radiated coefficients cata be quite different from the theoretical ones because of this effect. In this paper, a compensation rathod is presented allowing matching each elemejt from the array. The parameters of the array coupling model are obtained through the measurement of radiation pattern of the elements without the feeding network. An application to linear patch array is presented as an example

\section{INTRODUCTION}

Actual input impedance and radiated field from each element in the array can differ very much from the single element model. Input impedance can be computed through an N-port network model described by impedance $(Z)$ or scattering $(S)$ matrix. Radiated field call be computed if we know the active radiated field from each element that takes into account the complete array influence in each element radiated field as presented by account the complete array influence in each element radiated field, as presented by
Mailhoux [1]. In receiving anternas the tnode! would be the satnc, giving an equiva]ent active diagtam and active output impedance that depend on the loading cireuit. In large arrays, most elements present similar conditions: their class, position and load. That situation provides alrsost equal active impedance and diagram for most elements in the array except the extreme ones. In [2], Wasylkiwskij shows the relation between input impedance and radiateu fiejd for Minimum Scattering Antennas (MSA). Many times, real antennas have been assumed as MSA with good results [3]. In other cases, that assumption is not as fortunate and selation betwees input coupling matrix $(Z)$ and active radiated freld is not so clear. One of the most important questions is the design of antenna diagram or in the active and adaptive antenna behavious is if the coupling can be modelled by an $N x N$ coupling matrix $(C)$ as described in most studies of adaptive arrays [4].

In this paper, a matrix model is presented for array antennas fed through linear networks. The model is based in the several active modes of the array elements and active diagram computation as a function of these modes. If $k$ independent modes can describe the electromagtetic behaviout of each element, $N x k$ modes will be needed to define all the aray radiation characteristics. An $N x(k+l)$ square matrix will describe the array bebaviour. This description is independent of the feeding network, feeding distribution or transmission-receptson application [5]. For many printed patches only one resonant radjation mode can be used to describe the radiated field of each element. When this happens a $2 N \times 2 N$ mairix describes the corrplete aray model. Based on this model, and applying the feeding nework parameters, a coupling $C$ matrix can be obrained to compensate the design model from the influence of element coupling. The parameters of this model can be obained through the mcasurements of the radiation patten for ins iving individual element in the final array disposition. As an example, a printed anterna of
rectangular patches as that described by Pozar in [6], allows us to demonstrate how accurate the matrix model introduced in this paper is.

\section{RADIATEO/RECEIVE FIELD MODEL}

Transmission model for an individual antenna

The antenna electrical behaviour ean be defined by its input impedance and its radiated field (1): 


$$
\bar{E}_{\text {rad }}=v_{c} \dot{e}_{\theta}(\theta, \phi) F(\theta, \phi) \frac{\exp \left(-j k_{B} r\right)}{r}
$$

where $v_{E}$ is a voltage proportional to the input current. $F(\theta, \phi)$ is the radiation pattern, $\hat{\varepsilon}_{e}(\theta, \phi)$ is the polarisation vector. The antenna can also be seen as a function of its scattering matrix. Then the radiated field and the radiated power can be expressed as (2) and (3)

$$
\begin{aligned}
& \vec{E}_{r a d}(\theta, \phi)=b_{s} \sqrt{2 \eta_{0}} F(\theta, \phi) \frac{\exp \left(-j k_{0} j\right)}{r} \dot{e}_{c}(\theta, \phi) \\
& P_{r a d}=\eta\left|a_{i}\right|^{2}\left(l-\left.\left|S_{a}\right|^{2}|=| b_{s}\right|^{2} \int_{d \pi} \mid F_{s}\left(\theta,\left.\phi\right|^{2} d \Omega=\left|b_{r}\right|^{2}\right.\right.
\end{aligned}
$$

$S_{p}$ represents the reflection coefficient at the input defined respect $Z_{m}, b_{e}$ is a power wave proportional to the atnplitude and phase of the imput power wave (ari). The input reference impedance is $Z_{0}$ while the output reference impedance is $\eta_{0}=120 \pi$. The antenria gain can be expressed using the normalised radiation pattern and the antenna efficiescy as (4)

$$
G(\theta, \phi)=\eta 4 \pi \mid F\left[\left.\theta_{0} \phi \phi^{2}\right|^{2}=\frac{\left|S_{t}\right|^{2}}{1-\left|S_{0}\right|^{2}} 4 \pi|F(\theta, \phi)|^{2}\right.
$$

\section{Reception model for an individual antenna}

When the antenna works in receiving way the equivalent surface $A_{-}(\theta, 0)$ (5) reptesents the amount of power taken by the antejna. If $S_{p}$ represents reflection coefficient of the circuit, then a power wave $\left(b_{0}\right)$ can be extracted at the input port.

$$
A_{2}(\theta, \phi)=\frac{\lambda^{2}}{4 \pi}-G(\theta, \phi)=\eta \lambda^{2} \mid F(\theta, \phi)^{2}
$$

$$
P_{\mathrm{dis}}=\left|\mathrm{a}_{\mathrm{e}}\right|^{2} \frac{\left|\mathrm{S}_{\mathrm{r}}\right|^{2}}{1-\left|\mathrm{S}_{\mathrm{i}}\right|^{2}}=\left.\left|\hat{\hat{\mathrm{e}}}_{\mathrm{i}} \cdot \hat{\mathrm{e}}_{\mathrm{e}}(\theta, \phi)^{2} \frac{1}{2 \eta_{\mathrm{o}}}\right| E_{\mathrm{i}}\right|^{2} \frac{\left|\mathrm{S}_{\mathrm{r}}\right|^{2}}{1-\left|\mathrm{S}_{\mathrm{l}}\right|^{2}} \lambda^{2} \mid \mathrm{F}\left(\theta,\left.\phi\right|^{2}\right.
$$

The reception power wave proportional to the impinging field is (7):

$$
a_{z}=\left[\hat{e}_{i} \cdot \hat{e}_{c}(\theta, \phi)\right]-\frac{1}{\sqrt{2 \eta_{0}}} \cdot E_{i}|\lambda| F(\theta, \phi)
$$

Finally, if the reciprocity principle is applied then $S_{e}=S_{r}$

\section{Radiated/received field model for an array antenna}

A first approach of a model to take into account the previous effects has been proposed in $[5,7]$. This new network takes into accoum the decomposition of the current distribution in multiple characteristic modes. Then, any $N$-array antenna can be represented as a $(k+1) \times N$-network. The new network has one input and $k$ output ports corresponding to any of the radiating modes of each antenta (by input ports we mean any of the actuat probes of the aray while by output ports we mean fictitious ports representing any radiating function). Figure 1 shows the new $1+n$ port network ( $l$ comesponding to the antenna input and $n$ to the radiation modes). When the radiating elements are resonant rnicrostrip anternas, only one radiating mode may be considered resulting in a $2 N$-port 
network. This network is represented in Figure 2. The terminals at the left side of the $2 N$ port network represent physical probes of the antetana that can be directly measured while the ones at the right side allow us to define the radiation functions. They will never be charged since they represent ideal radiattng $\left(b_{e}\right)$ or receiving $\left(a_{2}\right)$ antennas. Then the tratrix equation relating previous variables is given in (8)

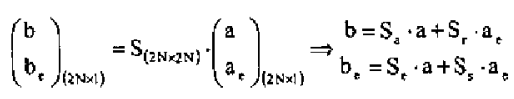

where $S_{a}$ represents the reflection coefficient, vector $S_{e}$ represents the tansmission

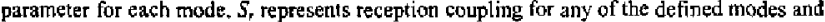
matrix $S_{s}$ indicates the scattered field by each node.

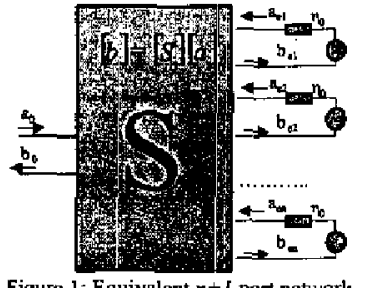

Figure 1: Equivalent $n+I$ port network for an individual antesna with sevęral radiation modes

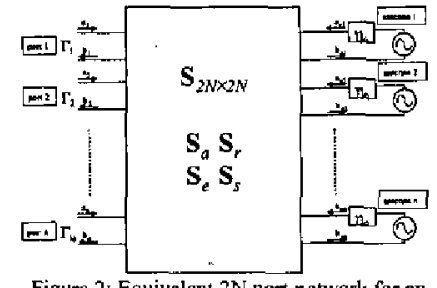

Figure 2: Equivalent 2N port network for an array antenna with only one radiation mode

When operating in a transrnitting way, the array is fed through a set of generators with an equivalent incident wave $a_{\mathrm{g}(N \times J)}$ and source reflection coefficient $\Gamma_{z\left(N \times N^{\prime}\right)}$. In this case equation (8) can be simplified, because $a_{r}=0$. The total radiated field can be expressed as the sum of the contributions from any of the radiating elements. This can be written in vector form as the dot product of the steering vector and the transmitting power wave (9). A coupling matrix $\left(C_{e}\right)$ can be defined $(10)$

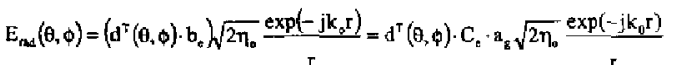

$$
\begin{aligned}
& C_{e}=S_{e} \cdot\left(I-\Gamma_{g} \cdot S_{a}\right)^{-1}
\end{aligned}
$$

$d$ is the steering vector in $(\theta, \phi)$ direction. Each term of matrix $C_{\varepsilon}\left(C_{\psi}\right)$ represents the amount of signal coupled from antenna $j$ to $i$. The radiation intensity can be expressed as the following dot product

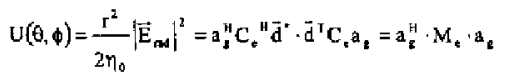

\section{MODEL COEFFICIENTES CALCULATION}

Once the model is defined, $S_{n}$ can be obtained through measurements with a vecror network analyser. $\Gamma_{\mathrm{g}}$ represents the generator reflection coefficient and $S_{e}$ matrix can be calculated through measurements of the radiation pattem for each individual antenna of the array,as follows

If a linear array of $\mathrm{N}$ independent patches (without feeding network) is measured exciting one patch each time (the elements of vector $a$ are equal to zero for all of them but 
onc), the colvmms of the matrix $S_{\mathrm{e}}$ (8) (considering $a_{e}=0$ ). $b_{e}$ is obtained after processing the radiation pattem. The ratiated field in a point of the space situated in far field respect every patch will be:

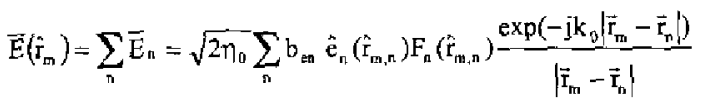

where the positions are shown in figure 3

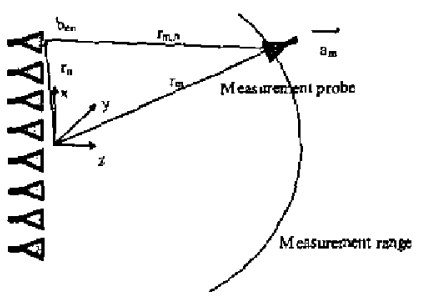

Figure 3: Dimensions in Measuremen structure

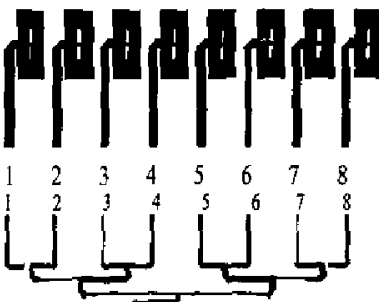

Figure 4: Application to design of the teeding network of a linear patch anay

The reception power is obtajned if measurement probe pattert $\left(F_{\mathrm{sm}}\right)$ is known (13) This equation represents the relation between the $b$, and the measured pattern, as wanted.

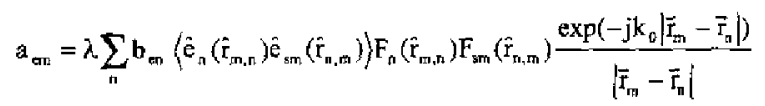

Normally, more points than elements are measured, so an optirnisationt process can be developed to obtain the coefficients $b_{k,}$ and the coupling matrix $C_{4}$. In [8] this procedure is followed to design the structure of figure 4 . Measurements of this structure will be presented at the conierence

\section{REFERENCES}

[1] J.R. Maillowx, Phased Aray Antenna Handibook. Artech House Ine. 1994

[2] W. Wasylkiwskyj and W. K. Kahn. Theory of Mutual Coupling Among MinimumSactieritig Antennas. IEEE Trans. on AP. Vol AP18, no.2, March 1970, pp. 204-216.

[3] 1.J. Gupta, A.A. Ksienski. Effect of Mutual Coupling on the Perfornance of Adaptive Arrays, IEEE Trans. on Arternas and Propagation, vol. AP-31, No 5 September 1983, pp 785-791.

[4] H. Steiskal and J.S. Herd Mutual Coupling Compensation in Small Array Antennas, IEEE Trans. on AP, Vol. AP-38 No. 12, December 1990, pp. 1971-1975

[5] D. Segovia-Vargas, R. Martín-Cuerda, M. Sierra-Pérez, Mutual Coupling Effects Correction in Microstrip Arrays for Dírection of Atrival (DOA) Estimation, IEE Proceedings on Microwave, Antentas and Propagation. 18th January 2002.

6] D.M. Pozar, Finite phased artay of rectangular microstrip patches. IEEE Trans on Antennas and propagation, Vol. AP-34, num. 5, May 86, pp. 658-665.

[7] M. Sierra-Pérez, D. Segovia-Vargas, M. Siema-Castañer, O. Moreno. Array Antenas Coupting Model for Multirnode radiators. Proc. of JINA, 2002. Nice, november, 2002.

[8] J.L. Masa, M. Sierra Pérez. Coupling Compensation in a Microstrip Patch Array. Proceedings of JINA, 2002. Niwe, novenber, 2002

'The simulations required have been done with CST Microwave Studio Software v-4.0, under a cooperation agreement between CST and Universidad Politéenica de Madrid. 